\title{
Performance of one-body reduced density matrix functionals for the homogeneous electron gas
}

\author{
N. N. Lathiotakis, ${ }^{1,2}$ N. Helbig, ${ }^{1,2,3,4}$ and E. K. U. Gross ${ }^{1,2}$ \\ ${ }^{1}$ Institut für Theoretische Physik, Freie Universität Berlin, \\ Arnimallee 14, D-14195 Berlin, Germany \\ ${ }^{2}$ European Theoretical Spectroscopy Facility \\ ${ }^{3}$ Fritz-Haber-Institut der Max-Planck-Gesellschaft, \\ Faradayweg 4-6, 14195 Berlin, Germany \\ ${ }^{4}$ Unité de Physico-Chimie et de Physique des Materiéaux, \\ Université Catholique de Louvain, B-1348 Louvain-la-Neuve, Belgium
}

\begin{abstract}
The subject of this study is the exchange-correlation-energy functional of reduced density matrix functional theory. Approximations of this functional are tested by applying them to the homogeneous electron gas. We find that two approximations recently proposed by Gritsenko, Pernal, and Baerends, J. Chem. Phys., 122, 204102 (2005), yield considerably better correlation energies and momentum distributions than previously known functionals. We introduce modifications to these functionals which, by construction, reproduce the exact correlation energy of the homogeneous electron gas.
\end{abstract}

PACS numbers: 71.10.-w,71.10.Ca,05.30.Fk,31.25.-v 


\section{INTRODUCTION}

Reduced-density-matrix-functional theory (RDMFT) is one possible way to tackle the problem of electronic correlation. It is based on Gilbert's theorem [1], which is an extension of the Hohenberg-Kohn theorem to non-local external potentials. Gilbert's theorem guarantees that the ground-state expectation value of any observable of a quantum mechanical system is a unique functional of the ground-state one-body reduced-density matrix (1-RDM). Thus, the fundamental quantity in RDMFT is the 1-RDM instead of the electronic density on which DFT is built upon. The properties of the exact 1-RDM have been the subject of theoretical studies for a long time [1, 2, 3, 4]. Nevertheless, only relatively recently approximate totalenergy functionals of the 1-RDM were used in practical applications [5, 6, 7, ㅇ, 9, 10, 11, 12, 13, 14, 15, 16, 17]. All these approximate functionals give a satisfactory account of electronic correlations in small atoms and molecules at the equilibrium distance. The latest generation of functionals performs equally well at the molecular dissociation limit. Especially this latest success makes RDMFT particularly appealing.

A great advantage of RDMFT, compared to DFT, is that the exact many-body kinetic energy is easily expressed in terms of the 1-RDM. More specifically, the total energy of a many-electron system in its ground state, characterized by an external potential $V(\mathbf{r})$, can be expressed in terms of the ground-state 1-RDM, $\gamma$ as

$$
\begin{aligned}
& E_{\text {tot }}[\gamma]=E_{\text {ion }}+\int d^{3} \mathbf{r} \int d^{3} \mathbf{r}^{\prime} \delta\left(\mathbf{r}-\mathbf{r}^{\prime}\right)[-\left.\frac{1}{2} \nabla_{\mathbf{r}}^{2}\right] \gamma\left(\mathbf{r}, \mathbf{r}^{\prime}\right) \\
&+\int d^{3} \mathbf{r} \int d^{3} \mathbf{r}^{\prime} \delta\left(\mathbf{r}-\mathbf{r}^{\prime}\right) V(\mathbf{r}) \gamma\left(\mathbf{r}, \mathbf{r}^{\prime}\right) \\
&+\frac{1}{2} \int d^{3} \mathbf{r} \int d^{3} \mathbf{r}^{\prime} \frac{\gamma(\mathbf{r}, \mathbf{r}) \gamma\left(\mathbf{r}^{\prime}, \mathbf{r}^{\prime}\right)}{\left|\mathbf{r}-\mathbf{r}^{\prime}\right|}+E_{\mathrm{xc}}[\gamma] .
\end{aligned}
$$

The first term in the above expression is a constant not related to the electronic degrees of freedom, for example the ion-ion repulsion energy. The next three terms are, respectively, the kinetic, the external potential and the electrostatic energy and they are known, explicit functionals of $\gamma$. Finally, the last term is the exchange and correlation (xc) energy which contains all the remaining electronic contributions to the total energy. The exact form of this functional is unknown and for practical applications needs to be approximated. Most of the approximate functionals for the xc energy that have been introduced so far are implicit functionals of $\gamma$. They depend explicitly on the natural orbitals $\varphi_{j}$, and the corresponding 
occupation numbers $n_{j}$ which are defined as the eigenfunctions and eigenvalues of the 1RDM:

$$
\int d^{3} \mathbf{r}^{\prime} \gamma\left(\mathbf{r}, \mathbf{r}^{\prime}\right) \varphi_{j}\left(\mathbf{r}^{\prime}\right)=n_{j} \varphi_{j}(\mathbf{r})
$$

Viewed as a functional of arbitrary $\gamma$ in an appropriately defined domain, the functional given in Eq. (11) has a minimum value at the ground-state $\gamma$. This appropriate domain is defined through subsidiary conditions for $\gamma$ known as $N$-representability conditions. It was shown by Coleman [18] that there are two such conditions for $\gamma$, and they concern the occupation numbers

$$
\sum_{j=1}^{\infty} n_{j}=N, \quad 0 \leq n_{j} \leq 1,
$$

where $N$ is the total number of electrons. These conditions guarantee that $\gamma$ corresponds to either a pure many-body state or an ensemble of pure states. The first condition can be enforced in the minimization with respect to $\gamma$ through the Lagrange-multipliers method. In that way, the quantity to be minimized becomes

$$
F=E_{\text {tot }}-\mu\left(\sum_{j=1}^{\infty} n_{j}-N\right),
$$

where $\mu$ is the corresponding Lagrange multiplier. $\mu$ was shown to be equal to the chemical potential, i.e. the derivative of the total energy with respect to the total number of electrons [19]. Interestingly, this allows one to exploit the discontinuity of $\mu$ as a function of the particle number for the calculation of the fundamental gap of materials and is a motivation for the development of RDMFT schemes for periodic systems in order to address questions like the semiconductor and insulator gaps.

The second of the $N$-representability conditions (3) has a dramatic consequence: it allows for border minima in the occupation number optimization. In other words, it allows for the the existence of a subset of the optimal occupation numbers which are exactly equal to either one or zero and do not satisfy the condition $\partial F / \partial n_{j}=0$. We refer to the corresponding states as pinned states. It is rather unlikely for the exact theory to produce pinned states for most systems of interest. For an occupation number being exactly equal to one, the corresponding natural orbital would be present in all determinants of the full CI expansion with non-zero coefficients. A situation like that has not been found for small atoms and molecules or for the HEG, where the exact solution can be calculated. While this is true for the exact xc functional, all the approximate functionals which are considered in this 
work, yield pinned states with $n_{j}=1$ for all systems we applied them to, except for the two-electron systems. These pinned states are core states and, in the exact theory, they correspond to occupation numbers which are marginally smaller than one. Hence, as far as the optimal $\gamma$ is concerned, the approximate result, $n_{j}=1$, is perfectly satisfactory. The important implication, however, is that $\partial F / \partial n_{j} \neq 0$ for the pinned states, and consequently $\delta F / \delta \gamma\left(\mathbf{r}, \mathbf{r}^{\prime}\right) \neq 0$ at the optimal $\gamma$.

A number of approximate functionals for $E_{\mathrm{xc}}$, including those of interest in the present work, can be cast into the form

$$
E_{\mathrm{xc}}[\gamma]=E_{\mathrm{xc}}\left[\left\{n_{j}\right\},\left\{\varphi_{j}\right\}\right]=-\frac{1}{2} \sum_{j, l=1}^{\infty} \int d^{3} \mathbf{r} \int d^{3} \mathbf{r}^{\prime} f\left(n_{j}, n_{l}\right) \frac{\varphi_{j}^{*}(\mathbf{r}) \varphi_{l}^{*}\left(\mathbf{r}^{\prime}\right) \varphi_{l}(\mathbf{r}) \varphi_{j}\left(\mathbf{r}^{\prime}\right)}{\left|\mathbf{r}-\mathbf{r}^{\prime}\right|}
$$

i.e. they have the form of the usual Hartree-Fock exchange modified by the function $f\left(n_{j}, n_{l}\right)$ of the occupation numbers. The first such approximation was introduced by Müller [4] and corresponds to the function $f\left(n_{j}, n_{l}\right)=\sqrt{n_{j} n_{l}}$. Müller considered a more general exponent for the occupation number product in the exchange-like term and found an optimal exponent of 1/2. By modelling the exchange and correlation hole, Buijse and Baerends [5] arrived at the same functional. Goedecker and Umrigar [6] (GU) considered a modification by explicitly removing the self-interaction (SI) terms. They also presented [6] a direct minimization with respect to the natural orbitals and the occupation numbers and found correlation energies for small atomic systems which are in very good agreement with the exact results. Later however, it was realized that the GU functional fails to reproduce the correct dissociation limit for small molecules [20, 21]. On the other hand, the Müller functional yields the correct dissociation limit but, in all cases, overestimates substantially the correlation energy [20, 21]. In the last decade, several other functionals of the 1-RDM have been introduced [8, 9, 10, 11, 12, 13, 14, 15, 16, 17] and applied to atomic and molecular systems. Recently, Gritsenko et al. [13] proposed improved 1-RDM functionals based on a hierarchy of repulsive corrections to the Müller functional. In that way, they attempted to correct the overcorrelation of this functional. The functionals corresponding to these hierarchical corrections, are called BBC1, $\mathrm{BBC} 2$, and $\mathrm{BBC} 3$. For all these functionals, it is essential to divide the natural orbitals into strongly and weakly occupied ones. This distinction appears naturally for finite systems since usually a subset of the orbitals corresponds to occupation numbers close to one, and the rest to occupation numbers close to zero. For the $\mathrm{BBC} 1$ and $\mathrm{BBC} 2$ functionals, the 
function $f\left(n_{j}, n_{l}\right)$ is:

$$
\begin{gathered}
\text { BBC1: } f\left(n_{j}, n_{l}\right)=\left\{\begin{array}{cl}
-\sqrt{n_{j} n_{l}}, & j \neq l, \text { and } j, l \text { weakly occupied, } \\
\sqrt{n_{j} n_{l}}, & \text { otherwise, }
\end{array}\right. \\
\text { BBC2: } \quad f\left(n_{j}, n_{l}\right)=\left\{\begin{array}{cl}
-\sqrt{n_{j} n_{l}}, & j \neq l, \text { and } j, l \text { weakly occupied, } \\
n_{j} n_{l}, & j \neq l, \text { and } j, l \text { strongly occupied, } \\
\sqrt{n_{j} n_{l}}, & \text { otherwise. }
\end{array}\right.
\end{gathered}
$$

Finally, in the BBC3 functional the anti-bonding orbital is treated as strongly occupied orbital. Additionally, the self-interaction terms are removed as in the GU functional, except for the bonding and anti-bonding orbitals. Gritsenko et al. [13] applied the BBC functionals to diatomic molecules and showed that they give an accurate description of these molecules at both the equilibrium distance and the dissociation limit.

There is a strong motivation for the extension and application of 1-RDM functionals to solid-state systems. This motivation stems from the success of these functionals in the description of electron correlation for finite systems, as well as the difficulties of DFT methods in describing certain materials and properties such as the band gap of semiconductors and insulators [22, 23, 24, 25] or the band width of the conduction band in $\mathrm{Na}[26]$.

A very important prototype system, which serves as a benchmark for the performance of approximate 1-RDM functionals when applied to periodic systems, is the homogeneous electron gas (HEG). Furthermore, this system can serve as a laboratory for the development of approximate functionals, in a fashion similar to DFT. As a consequence of translational invariance, the natural orbitals can be chosen as plane waves and the search for the ground state 1-RDM is restricted to the optimization of the momentum distribution $n(\mathbf{k})$, which is the occupation number that corresponds to the plane-wave natural orbital with wavevector k. An important point to note is that the self-interaction terms for the plane-wave natural orbitals vanish. Consequently, the GU and the Müller functionals are identical. Finally, approximations that involve a special treatment of single orbitals have zero effect in the continuous wavevector case. Thus, the BBC3 functional is identical to BBC2.

As a consequence of the rotational invariance of the HEG the occupation numbers have the property $n(\mathbf{k})=n(k)$, i.e. all the plane-wave natural orbitals corresponding to the same absolute value $k$ are degenerate (with respect to the occupation number). This allows one to perform unitary transformations among the degenerate plane waves leading, e.g., to 
angular-momentum eigenfunctions

$$
\varphi_{k l m}(\mathbf{r})=j_{l}(k r) Y_{l m}(\Omega)
$$

for the natural orbitals, where $j_{l}(k r)$ are spherical Bessel functions and $Y_{l m}$ spherical harmonics. Since these functions are localized in real space it is conceivable to include selfinteraction corrections in terms of the natural orbitals (8) for the HEG. To our knowledge, this possibility has not been explored so far and is also beyond the aim of the present work.

Choosing the natural orbitals as plane waves, the 1-RDM of the HEG can be written as

$$
\gamma\left(\mathbf{r}, \mathbf{r}^{\prime}\right)=\frac{2}{V} \sum_{\mathbf{k}} n(k) e^{i \mathbf{k}\left(\mathbf{r}-\mathbf{r}^{\prime}\right)}
$$

where $V$ is the volume of the system $(V \rightarrow \infty)$. Substituting this expression in Eqs. (1) and (5) we obtain for the total energy

$$
E_{\text {tot }}=2 \sum_{\mathbf{k}_{1}} \frac{\mathbf{k}_{1}^{2}}{2} n\left(k_{1}\right)-\frac{1}{V} \sum_{\mathbf{k}_{1}, \mathbf{k}_{2}} f\left(n\left(k_{1}\right), n\left(k_{2}\right)\right) \frac{4 \pi}{\left|\mathbf{k}_{1}-\mathbf{k}_{2}\right|^{2}+\alpha^{2}},
$$

where $\mathbf{k}_{1}, \mathbf{k}_{2}$ are wavevector indices, and $\alpha$ is a small quantity (usually $\sim 10^{-8}$ ) included for numerical stability. As in Hartree-Fock, the external potential energy and the electronic Coulomb repulsion energy, i.e. the third and fourth terms in Eq. (11) cancel exactly with the first term i.e. the ion-ion interaction. The quantity $F$ of Eq. (4) per particle then becomes

$$
\begin{aligned}
& \frac{F}{N}=\frac{3}{2 k_{\mathrm{F}}^{3}} \int_{0}^{\infty} d k_{1} k_{1}^{2}\left(k_{1}^{2}-2 \mu\right) n\left(k_{1}\right) \\
& \quad+\frac{3}{4 \pi k_{\mathrm{F}}^{3}} \int_{0}^{\infty} d k_{1} \int_{0}^{\infty} d k_{2} k_{1} k_{2} \log \left[\frac{\left(k_{1}-k_{2}\right)^{2}+\alpha^{2}}{\left(k_{1}+k_{2}\right)^{2}+\alpha^{2}}\right] f\left(n\left(k_{1}\right), n\left(k_{2}\right)\right)+\mu,
\end{aligned}
$$

where $k_{\mathrm{F}}=(9 \pi / 4)^{1 / 3} r_{s}^{-1}$ is the Fermi-wavevector of the non-interacting HEG and $r_{s}$ is the radius (in atomic units) of the sphere with volume equal to the volume per electron.

Cioslowski and Pernal [27] applied the Müller functional to the HEG and calculated analytically the resulting momentum distribution

$$
n(k)=512 \pi \rho\left(1+4 k^{2}\right)^{-4},
$$

where $\rho$ is the electron-density-per-spin, $\rho=3\left(8 \pi r_{s}^{3}\right)^{-1}$. The corresponding total energy per particle is independent of the density and equal to $-1 / 8$ Hartree. It is obvious that the solution of the Eq. (12) is consistent with the second $N$-representability constraint of Eq. (3) 
only for $\rho \leq(512 \pi)^{-1}$, i.e. $r_{s} \geq 5.77$. In other words, the Müller functional gives a solution without pinned states only for $r_{s} \geq 5.77$. In addition, Cioslowski and Pernal demonstrated that the Oxford-Lieb [28] bound is violated for $\rho \geq 1.65 \times 10^{-3}$, i.e. $r_{s} \leq 4.167$. The solution with pinned states for $r_{s}<5.77$, was calculated by Csányi and Arias [7]. More specifically, for $r_{s}<5.77$, one gets an optimal momentum distribution $n(k)$ with $n(k)=1$ for $k$ below a certain value $k_{p}$ and fractional $n(k)<1$ for $k>k_{p}$. This behavior is in complete analogy to the case of finite systems for the Müller functional. Unfortunately, it is in conflict with the fact that the exact momentum distribution [29, 30] is a monotonically decreasing function of $k$ and is strictly smaller than 1, i.e. there are no pinned states. Additionally, the exact momentum distribution is concave for $k<k_{\mathrm{F}}$, it shows a discontinuity at $k_{\mathrm{F}}$, and for $k>k_{\mathrm{F}}$ it goes to zero asymptotically. The size of the discontinuity is decreasing with $r_{s}$.

In addition to the Müller functional, Csányi and Arias [7] considered a similar functional derived from a tensor product expansion of the two-body density matrix, which they called Corrected Hartree-Fock (CHF). Unfortunately, CHF gives zero correlation for the HEG in the high-density limit $\left(r_{s} \rightarrow 0\right)$, coinciding with Hartree-Fock. In the opposite limit, it strongly overcorrelates giving the same results as the Müller functional. In the intermediate region, including the metallic densities, the result for the correlation energy is close to the exact but its dependence on $r_{s}$ is monotonically decreasing instead of increasing. In an attempt to improve over the Müller functional and CHF, Csányi, Goedecker and Arias considered an improved tensor product expansion of the two-particle density matrix [8]. The resulting functional, which is called CGA, performs very well in the high-density regime and significantly better than the previous two functionals in the region of metallic densities. The deviation from the exact correlation energy increases with $r_{s}$ and at higher densities CGA coincides with $\mathrm{CHF}$ and the Müller functional.

In the present work, we apply the $\mathrm{BBC} 1$ and $\mathrm{BBC} 2$ functionals of Gritsenko et al. [13] to the HEG and compare with previous functionals as far as the resulting correlation energies are concerned. We also investigate other features like the resemblance of the resulting momentum distribution to the exact and the state-pinning. In order to apply the $\mathrm{BBC} 1,2$ functionals to the case of the HEG through Eq. (11), we need to distinguish between strongly and weakly occupied orbitals. For finite systems, Gritsenko et al. [13] chose the first N/2 natural orbitals to be strongly occupied. In complete analogy, we can use a critical wavevector $k_{c}=k_{\mathrm{F}}$ below which all states are assumed to be strongly occupied while above they are 
weakly occupied.

An additional goal of the present work is to demonstrate that the HEG can be used to develop functionals suitable for metallic systems. The idea is to modify the BBC1 functional in such a way that it yields the exact correlation energy for the HEG. This is achieved in two different ways: (i) For each given density we choose $k_{c}$ such that BBC1 reproduces the exact correlation energy of the HEG at that density. We call this functional $k_{c}$-functional. (ii) We introduce a function $s\left(r_{s}\right)$ multiplying the xc terms of Eq. (15) for two weakly occupied orbitals, keeping $k_{c}=k_{\mathrm{F}}$. In this way, we replace the sign change of the BBC1 functional with the parameter $s$. Accordingly, we call this functional $s$-functional.

In the following section we present details of the numerical implementation as well as the results of applying the BBCs and the $k_{c^{-}}$and $s$-functional to the HEG.

\section{NUMERICAL IMPLEMENTATION, RESULTS}

The minimization of the energy expression (11) with respect to $n(k)$ is performed using the steepest-descent method. We choose to work in energy-space instead of $k$-space, i.e. we perform the variable substitution $\epsilon=k^{2} / 2$ and solve numerically the minimization problem for $n(\epsilon)$. Working in energy-space rather than $k$-space improves the stability of the numerical treatment. The energy $\epsilon$ is discretized using a double-logarithmic mesh centered at $\epsilon_{\mathrm{F}}=$ $k_{\mathrm{F}}^{2} / 2$, where the occupation varies the most. The upper limit of integration is chosen such that the momentum-distribution function has dropped to values smaller than $10^{-6}$. The double integration with respect to the energy is carried out using an adaptive grid technique capable of treating integrable singularities like the logarithmic singularity of the present problem. The values of the energy-distribution function $n(\epsilon)$ in between the mesh points, necessary for the adaptive grid method, are obtained from an interpolation scheme. The $N$-representability constraint $0 \leq n(\epsilon) \leq 1$ is implemented through the substitution $n(\epsilon)=$ $\sin ^{2}[\pi \theta(\epsilon) / 2]$ and variation with respect to $\theta(\epsilon)$. Extra care is required to avoid $n(\epsilon)$ being falsely pinned to 0 or 1 . Indeed, if for a particular $\epsilon, n(\epsilon)$ gets very close to 1 or 0 during the variation it would stay pinned at that point. The variation with respect to the Lagrangemultiplier $\mu$ is implemented as an external iterative procedure, thus achieving convergence for each value of $\mu$. The correct value of $\mu$ is selected by requiring the momentum-distribution

function to integrate to the correct number of electrons for a given value of $r_{s}$. Finally, we 


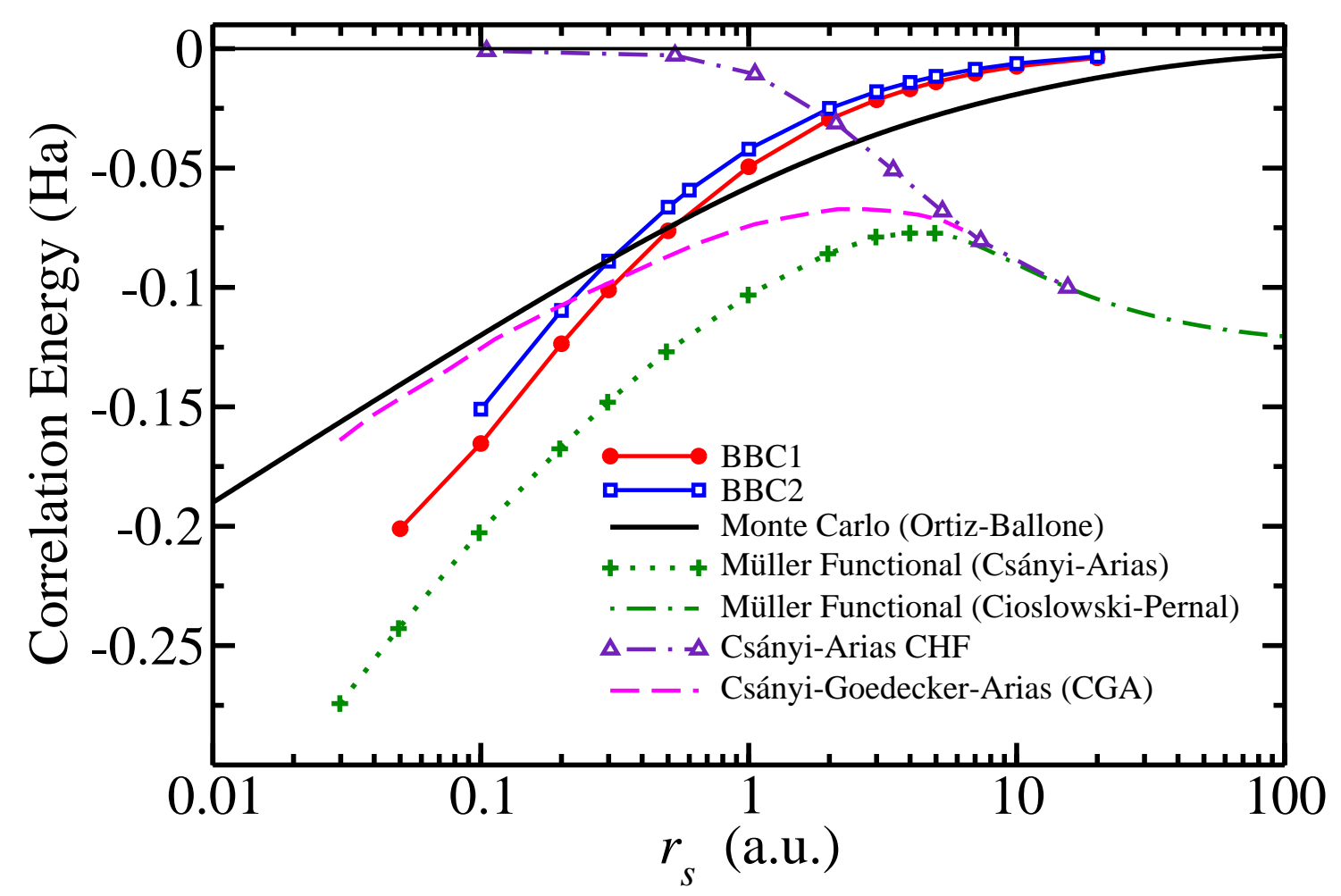

FIG. 1: (Color online) The correlation energy of the HEG as a function of $r_{s}$ calculated with the BBC1 and BBC2 functionals compared to various other calculations. The Monte Carlo result represents the Perdew-Wang fit [31] of the DMC data of Ortiz and Ballone [29]. The dotted line corresponds to the numerical results by Csányi and Arias [7] employing the Müller functional, for $r_{s}<5.77$. Its continuation, the dash-dotted line, for $r_{s}>5.77$, stands for the analytical results of Cioslowski and Pernal [27] employing the same functional. The results for the CHF functional [7] as well as the CGA [8] are also shown.

found that a reasonable value for the parameter $\alpha$ in Eqs. (10) and (11) is $10^{-8}$, which avoids both numerical problems as well as the dependence of the results on $\alpha$.

\section{A. Application of the BBC functionals to the HEG}

In Fig. 1, we show the correlation energy of the HEG as a function of $r_{s}$. The correlation energy calculated with the $\mathrm{BBC} 1$ and $\mathrm{BBC} 2$ functionals is significantly closer to the exact than any other functional over the whole range of $r_{s}$. Both functionals also seem to reproduce the correct asymptotic limit of zero correlation for the dilute HEG. For small densities up to metallic densities, the $\mathrm{BBC}$ functionals under-correlate, i.e. the absolute value of the 
correlation energy is too small. In the dense limit, they over-correlate and the crossover is at around $r_{s}=0.5$ and 0.3 for the $\mathrm{BBC} 1$ and $\mathrm{BBC} 2$, respectively. Unfortunately, in the area of low metallic densities both functionals yield correlation energies which deviate from the exact values by $50 \%$. In absolute numbers, the error of BBC1 and BBC2 is of the same order as the RPA result [31]. Nevertheless, in the range $0.1<r_{s}<1$, the BBC functionals perform remarkably well. Compared to all previous 1-RDM functionals, BBC1 and BBC2 offer a much better account of the electron correlation for the HEG. Although less accurate than the CGA in the high density region, they perform better for metallic densities and they reproduce the limit of zero correlation at the dilute HEG limit where the Müller functional, CHF and CGA fail.

A feature of the exact momentum distribution, namely the discontinuity at the Fermi wavevector $k_{\mathrm{F}}$, is reproduced by the BBC functionals. The discontinuity is more pronounced for the BBC1 functional, as can be seen in Fig. 2, where we plotted the momentum distribution of the HEG with $r_{s}=1$ and $r_{s}=5$ using the Müller functional as well as BBC1 and $\mathrm{BBC} 2$. Contrary to $\mathrm{BBC} 1$ and $\mathrm{BBC} 2$ the Müller functional does not yield a discontinuity. To our knowledge, there is no report of any other 1-RDM functional reproducing this feature of the HEG. To extract the size of the discontinuity quantitatively, we used two energy mesh points very close to $\epsilon_{\mathrm{F}}$ (at a distance of $\pm 10^{-8} \epsilon_{\mathrm{F}}$ ). In Fig. 3, we plot the size of the discontinuity $\Delta n$ as a function of $r_{s}$. As we see, it increases monotonically with $r_{s}$ and has the tendency to saturate for large $r_{s}$ for both $\mathrm{BBC} 1$ and $\mathrm{BBC} 2$. For $\mathrm{BBC} 2$ the discontinuity is substantially smaller than for BBC1 and it goes to zero at $0.6<r_{s}<0.7$. Both the size and the dependence on $r_{s}$ are in complete disagreement with the exact theory, where $\Delta n$ is substantially bigger and decreases with $r_{s}$ as one can see from the two fits to the DMC data [29, 30].

We now turn to the question of state pinning. As we see in Fig. 2, state pinning is a common feature of all the functionals we employed. To verify that the states are truly pinned, we plot the functional derivative $\delta F / \delta n(k)$ which is non-zero for pinned states. As one expects, the number of pinned states decreases with increasing $r_{s}$. We define a wavevector $k_{p}$ below which the corresponding states are pinned, i.e. $n(k)=1$ for $k<k_{p}$. In Fig. 4, we plot $k_{p}$ as a function of $r_{s}$ for the Müller and the two BBC functionals. For all three functionals, $k_{p}$ decreases monotonically with $r_{s}$. For the Müller functional our numerical calculation confirms the analytic result [27] that above a critical value of $r_{s}=5.77$ 

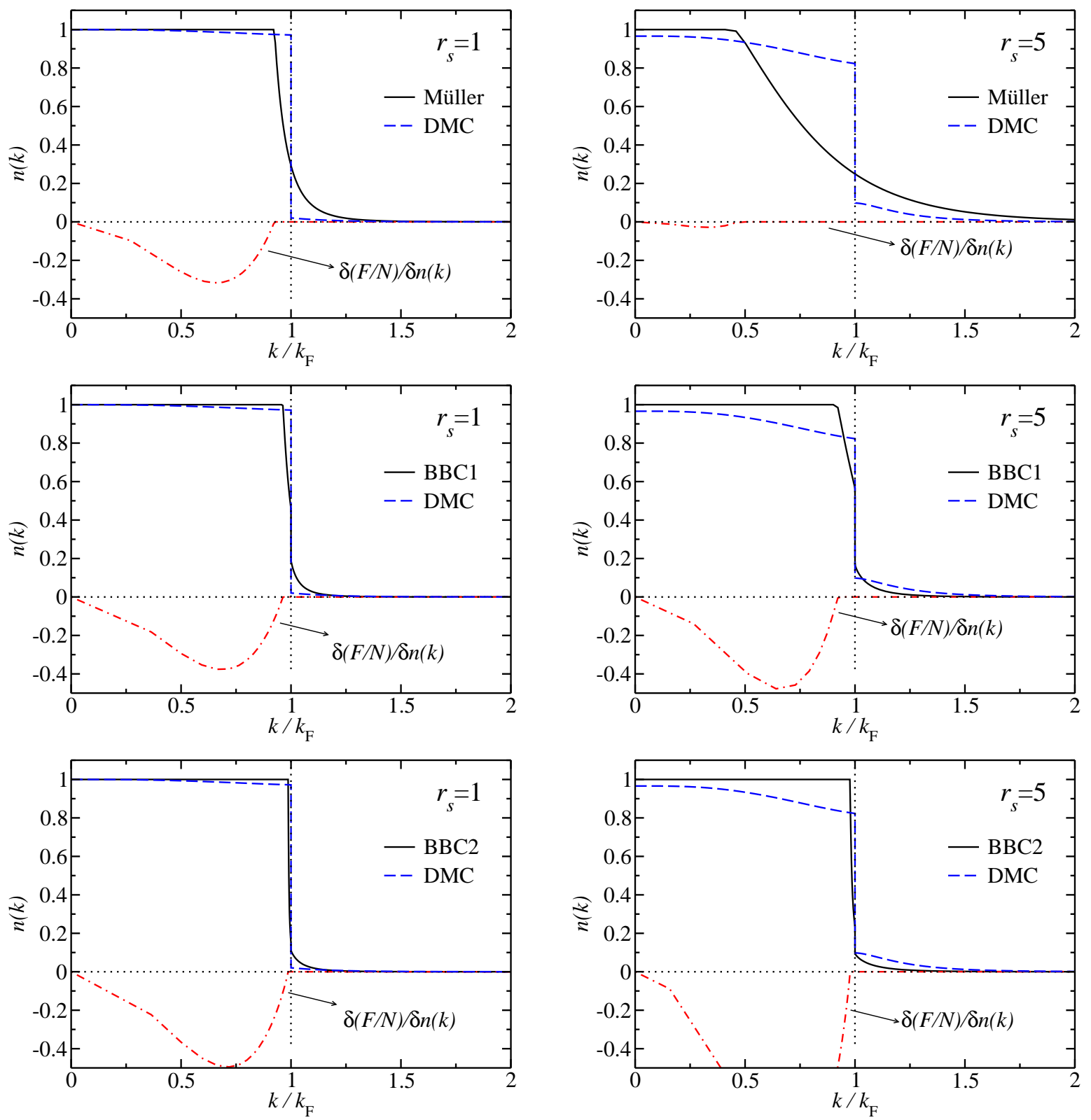

FIG. 2: (Color online) The momentum distribution $n(k)$ of the HEG for $r_{s}=1$ (left) and $r_{s}=$ 5 (right) calculated with the Müller functional, $\mathrm{BBC} 1$, and $\mathrm{BBC} 2 . \mathrm{BBC} 1$ and $\mathrm{BBC} 2$ show a discontinuity of the momentum distribution at $k_{\mathrm{F}}$. For comparison we include the fit to the DMC data of Ortiz-Ballone [29]. The derivative $\delta(F / N) / \delta n(k)$ is also plotted. Note that for small $k$ the derivative is not zero and $n(k)$ is pinned at one.

there are no pinned states, and therefore $k_{p}$ goes to zero at this value. This can already be seen in Fig. 2, where, for the Müller functional at $r_{s}=5$, the derivative is very close to zero even for small wavevectors. Interestingly, for BBC1 and $\mathrm{BBC} 2$ we found no such critical 


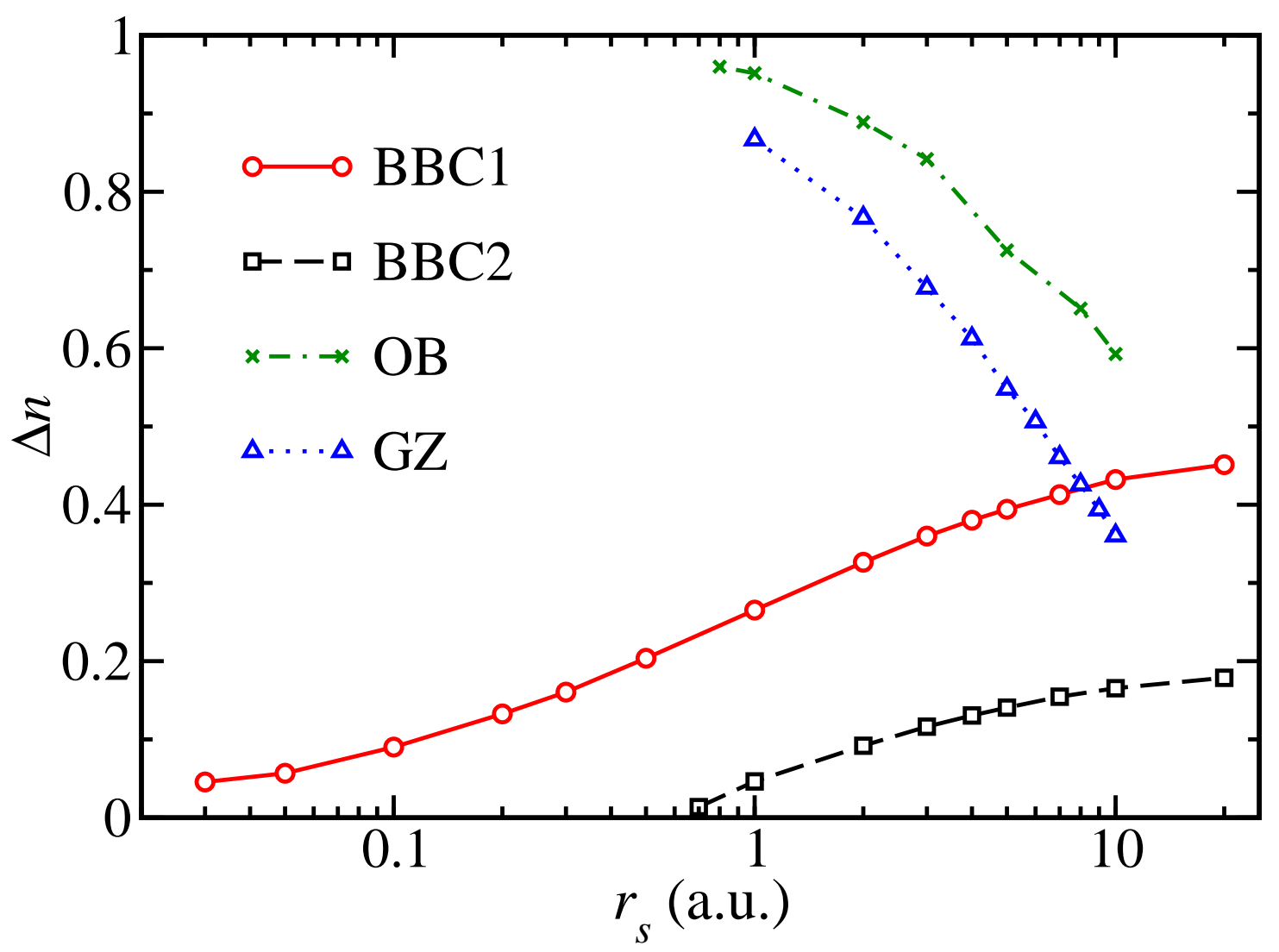

FIG. 3: (Color online) The discontinuity $\Delta n$ of the momentum distribution at $k_{\mathrm{F}}$ for $\mathrm{BBC} 1$ and $\mathrm{BBC} 2$ as a function of $r_{s}$, compared to the fits of Ortiz and Ballone (OB) [29], and Gori-Giorgi and Ziesche (GZ) [30] to the DMC data.

value up to $r_{s}=20$. Indeed, for both BBC1 and BBC2 the decrease of $k_{p}$ is much smaller than for the Müller functional, $k_{p}$ being almost constant for BBC2.

As we have seen, the performance of the BBC1,2 functionals for the HEG is improved significantly compared to previous functionals. This is especially remarkable, given that they were originally constructed to describe the dissociation of small molecules.

\section{B. Improved functionals for the HEG}

In this section, we attempt to improve over the BBC functionals for the HEG. We present two functionals which are simple modifications of the BBC1 functional. In both cases, the $\mathrm{BBC} 1$ functional is generalized by introducing an additional function of $r_{s}$ such that the correlation energy of the HEG is reproduced exactly for each $r_{s}$. As exact results we regard 


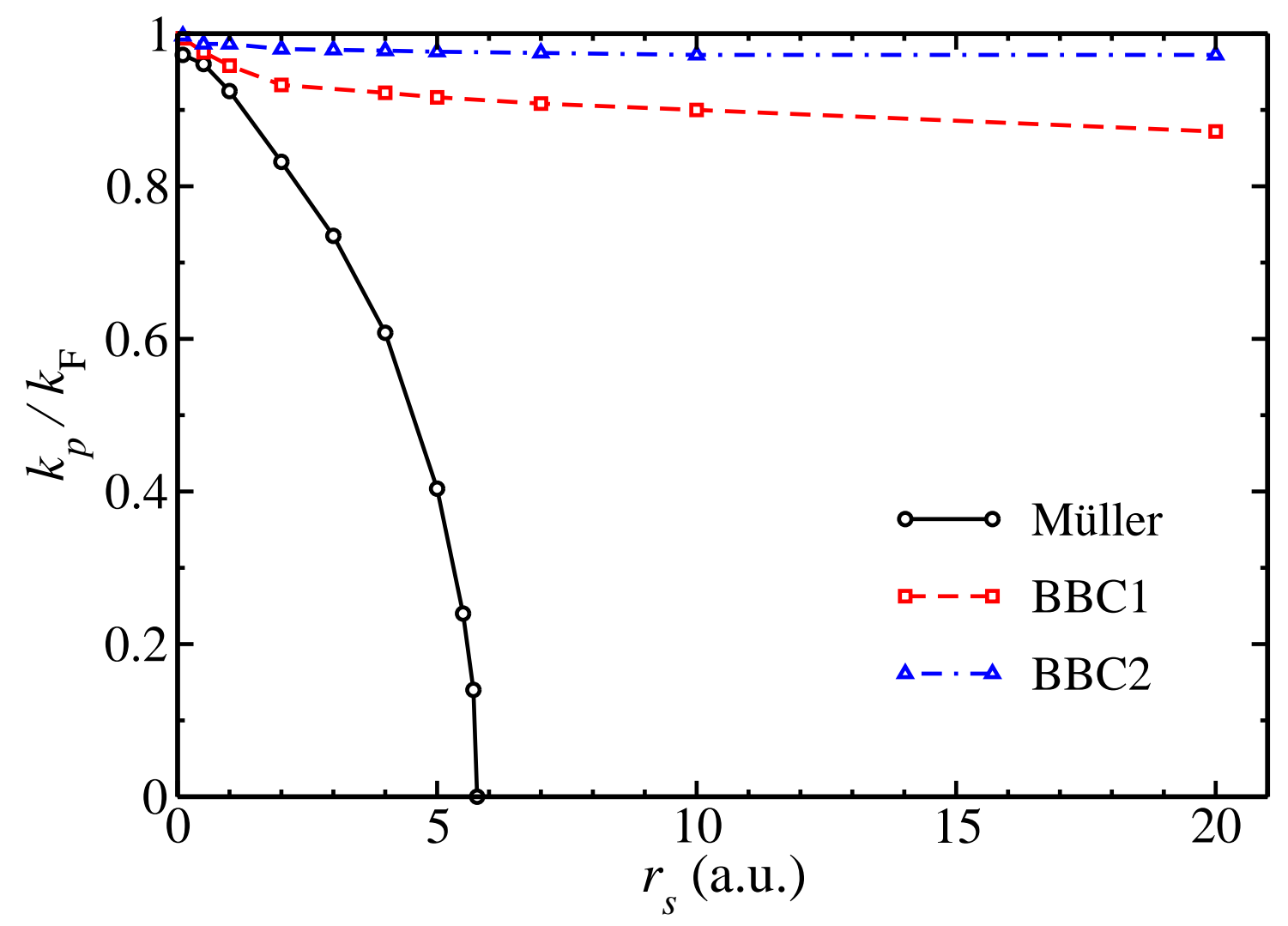

FIG. 4: (Color online) The wavevector $k_{p}$, where the optimal momentum distribution becomes fractional, as a function of $r_{s}$.

the Perdew-Wang fit 31] of the correlation energy obtained from diffusion Monte Carlo (DMC) calculations by Ceperley and Alder [32] and Ortiz and Ballone [29]. The two Monte Carlo calculations yield almost identical correlation energies.

For the first functional, we adjust the critical wavevector $k_{c}$, which is used to distinguish between strongly and weakly occupies states, instead of using $k_{c}=k_{\mathrm{F}}$, as in BBC1. We call this functional the $k_{c}$-functional. The corresponding function $f$ in Eq. (5) then reads

$$
f\left(n\left(k_{1}\right), n\left(k_{2}\right)\right)=\left\{\begin{aligned}
-\sqrt{n\left(k_{1}\right) n\left(k_{2}\right)}, & k_{1}, k_{2}>k_{c}\left(r_{s}\right), \\
\sqrt{n\left(k_{1}\right) n\left(k_{2}\right)}, & \text { otherwise. }
\end{aligned}\right.
$$

We perform the fitting of $k_{c}$ over the range of metallic densities, $0.5 \leq r_{s} \leq 5$. The results are compiled in Table I. For $r_{s}=0.5, k_{c} \approx k_{\mathrm{F}}$, since this point is almost exactly reproduced by BBC1 (see Fig. 1). For $r_{s}>0.5, k_{c}$ is a monotonically increasing function of $r_{s}$. Fitting $k_{c}$ has a strong impact on the momentum distribution which is displayed in Fig. 5 , for $r_{s}=1$ and $r_{s}=5$. It is not surprising that the discontinuity is displaced from $k_{\mathrm{F}}$ to $k_{c}$. 


\begin{tabular}{c||c|c|c|c|c|c}
\hline \hline$r_{s}$ & 0.5 & 1.0 & 2.0 & 3.0 & 4.0 & 5.0 \\
\hline$k_{c} / k_{\mathrm{F}}$ & 0.994 & 1.032 & 1.085 & 1.122 & 1.155 & 1.172 \\
\hline \hline
\end{tabular}

TABLE I: The the critical wavevector $k_{c}$ for some metallic densities.
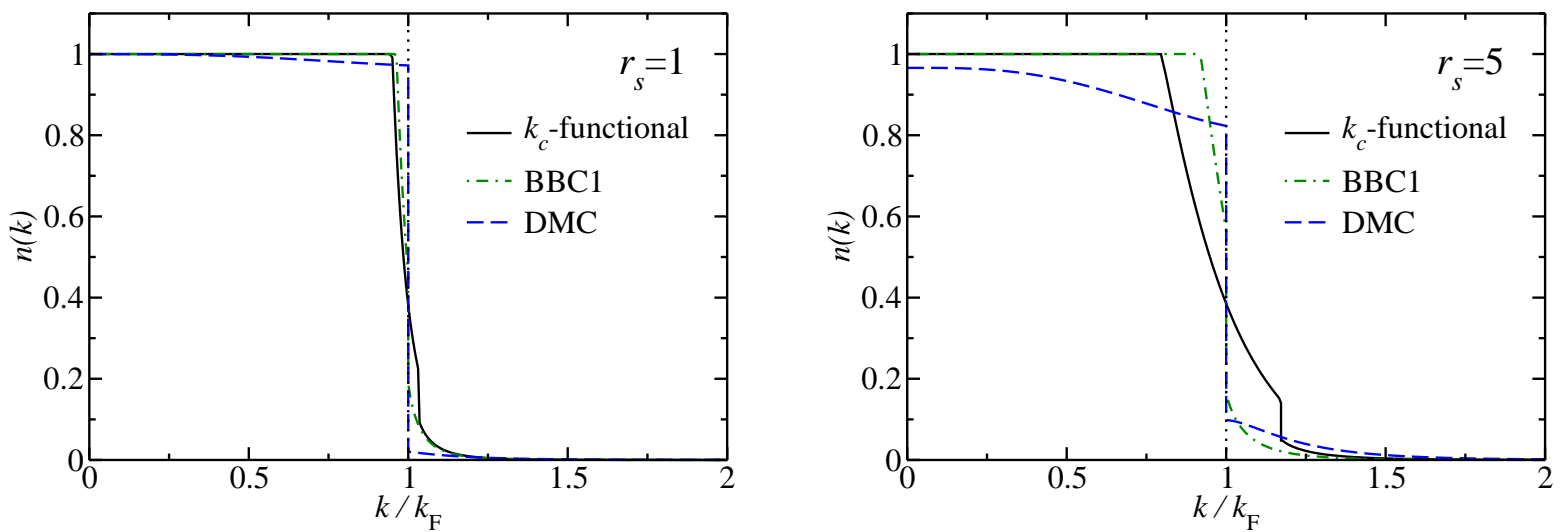

FIG. 5: (Color online) The momentum distribution for the $k_{c}$-functional compared with the BBC1 and the fit to the DMC data of Ortiz-Ballone [29], for $r_{s}=1$ and $r_{s}=5$. For the $k_{c}$-functional the discontinuity is moved from $k_{\mathrm{F}}$ to $k_{c}$, i.e to $1.032 k_{\mathrm{F}}$ and $1.172 k_{\mathrm{F}}$ respectively.

Additionally, its size is reduced significantly compared to BBC1. Both the displacement of the discontinuity and the decrease in the step size are in disagreement with the exact result.

An alternative idea is to keep $k_{c}=k_{\mathrm{F}}$ fixed and consider a fitting parameter $s$ multiplying the exchange-like terms when both states, $\mathbf{k}_{1}$ and $\mathbf{k}_{2}$, are weakly occupied, i.e.

$$
f\left(n\left(k_{1}\right), n\left(k_{2}\right)\right)=\left\{\begin{aligned}
-s\left(r_{s}\right) \sqrt{n\left(k_{1}\right) n\left(k_{2}\right)}, & k_{1}, k_{2}>k_{\mathrm{F}}, \\
\sqrt{n\left(k_{1}\right) n\left(k_{2}\right)}, & \text { otherwise. }
\end{aligned}\right.
$$

In this way, the parameter $s$ which is a kind of strength of the xc terms becomes a function of $r_{s}$, as indicated in Eq. (14). We call this functional the $s$-functional. The values of $s$ for the fitting to the Ortiz and Ballone DMC results are included in Table III. As we see, $s$ varies between 4 and -0.26 for the range of densities, $0.1 \leq r_{s} \leq 10$, we considered. In Fig. 6, we show the resulting, monotonically decreasing function $s\left(r_{s}\right)$.

From Fig. 7 one can see that one of the advantages of fitting $s$ instead of $k_{c}$ is that the discontinuity of the momentum distribution remains fixed at $k_{\mathrm{F}}$. In addition, its size is almost constant $(\approx 0.2)$ as a function of $r_{s}$. As we see in Fig. 3, the exact discontinuity is significantly higher and it is a decreasing function of $r_{s}$. Therefore, concerning the size of the discontinuity, the $s$-functional does not improve over the BBC1, which, in the dilute gas 


\begin{tabular}{ccccc}
\hline \hline$r_{s}$ & $\mathrm{~s}$ & & $r_{s}$ & $\mathrm{~s}$ \\
\cline { 4 - 4 } 0.1 & 4.913 & & 1.0 & 0.435 \\
0.2 & 2.751 & & 1.5 & 0.190 \\
0.3 & 1.867 & & 2.0 & 0.059 \\
0.4 & 1.390 & 3.0 & -0.074 \\
0.5 & 1.087 & & 4.0 & -0.146 \\
0.6 & 0.877 & 5.0 & -0.189 \\
0.7 & 0.727 & 7.0 & -0.234 \\
0.8 & 0.602 & 10.0 & -0.263 \\
\hline \hline
\end{tabular}

TABLE II: The fitted values of $s$ for various values of $r_{s}$ for the $s$-functional. $s$ was fitted to reproduce the correlation energies of the DMC calculation of Ortiz and Ballone [29].

limit, is close to the exact result. However, the increasing behavior of BBC1 is improved by the $s$-functional which yields discontinuities that are almost constant as a function of $r_{s}$.

In conclusion, the $s$-functional results in momentum distributions that resemble the exact ones over the whole range of $r_{s}$ more closely than any of the other functionals considered here.

\section{CONCLUSION}

We have applied a variety of 1-RDM functionals to the HEG. We show that the BBC functionals [13] yield a significant improvement over previous functionals as far as the correlation energy is concerned. In addition, they yield a discontinuity of the momentum distribution at the Fermi wavevector in resemblance of the exact HEG theory. However, the size and the dependence on the density of this discontinuity are not in agreement with the quantum Monte Carlo results.

By introducing an appropriately fitted function of $r_{s}$ in the BBC1 functional, we demonstrate that the exact correlation energy of the HEG can be reproduced with a smooth and monotonic fitting function. For this function, we either use the critical wavevector $k_{c}\left(r_{s}\right)$ which distinguishes between the strongly and weakly occupied states, or a strength $s\left(r_{s}\right)$ multiplying the exchange-like terms for two weakly occupied states. Both of these proce- 


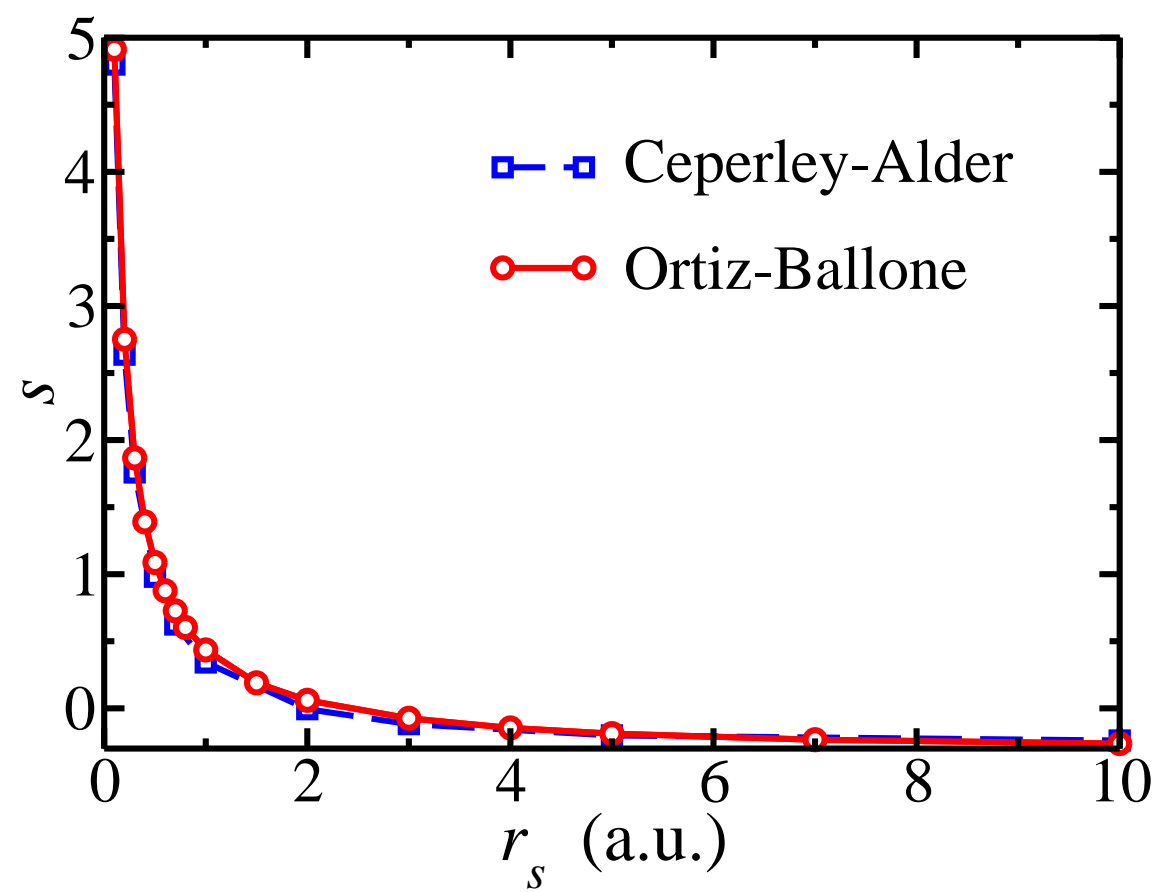

FIG. 6: (Color online) The dependence of the fitting parameter $s$ on $r_{s}$ resulting from fits to the Perdew-Wang parameterization [31] of the correlation energy from two different sets of DMC results: the Ceperley and Alder [32] and the Ortiz and Ballone [29].
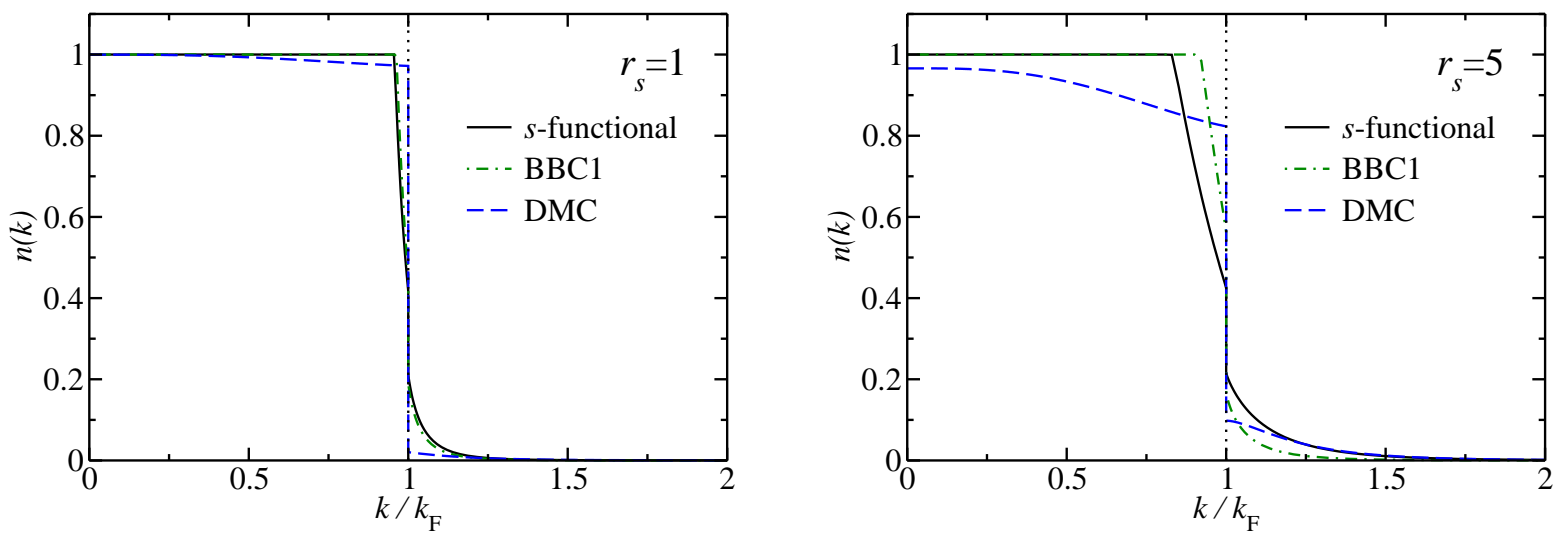

FIG. 7: (Color online) The momentum distribution given by the $s$-functional, compared with the BBC1 and the fit to the DMC data of Ortiz-Ballone [29], for $r_{s}=1(s=0.435)$, and $r_{s}=5$ $(s=-0.189)$. The discontinuity remains at $k_{\mathrm{F}}$ and is approximately equal to 0.2 .

dures were applied to the BBC1 functional. The two functionals, yielding by construction the exact correlation energy of the HEG, are assessed by the quality of the resulting momentum distributions. We show that choosing the second procedure, i.e. the $s$-functional, is superior to fitting $k_{c}$. The discontinuity $\Delta n$ of the momentum distribution resulting from 
the $s$-functional is nearly constant as a function of $r_{s}$ and hence represents a significant improvement over $\mathrm{BBC} 1$ and $\mathrm{BBC} 2$. However, the momentum distribution obtained by the $s$-functional still deviates significantly from the exact one. To remedy this, more complicated strategies have to be considered, possibly with the introduction of more fitting parameters.

Our functional of choice, being derived from the HEG, is expected to yield good results for metallic systems. The application to finite as well as non-metallic periodic systems is not straightforward because $r_{s}$ is not well-defined in these cases. Hence, the necessity arises to map $r_{s}$ onto other quantities characterizing these systems, or to involve a LDA-type prescription relating $s\left(r_{s}\right)$ to the local density.

\section{Acknowledgments}

This work was supported in part by the Deutsche Forschungsgemeischaft within the program SPP 1145, by the EXCITING Research and Training Network, and by the EU's Sixth Framework Program through the Nanoquanta Network of Excellence (NMP4-CT2004-500198).

[1] T. L. Gilbert, Phys. Rev. B12, 2111 (1975).

[2] S. M. Valone, J. Chem. Phys. 73, 1344 (1980); 73, 4653 (1980).

[3] G. Zumbach and K. Maschke, J. Chem. Phys. 82, 5604 (1985).

[4] A. M. K. Müller, Phys. Lett. A 105, 446 (1984).

[5] M. A. Buijse, PhD Thesis, Vrije Universiteit Amsterdam (1991); M. A. Buijse, E. J. Baerends, Mol. Phys. 100, 401 (2002).

[6] S. Goedecker, C. J. Umrigar, Phys. Rev. Lett. 81, 866 (1998).

[7] G. Csányi, T. A. Arias, Phys. Rev. B61 (2000) 7348.

[8] G. Csányi, S. Goedecker, T. A. Arias, Phys. Rev. A65 032510 (2002).

[9] K. Yasuda, Phys. Rev. Lett. 88, 053001 (2002).

[10] C. Kollmar, J. Chem. Phys. 121, 11581 (2004).

[11] J. Cioslowski, K. Pernal, M. Buchowiecki, J. Chem. Phys. 119, 6443 (2003).

[12] K. Pernal, J. Cioslowski, J. Chem. Phys. 120, 5987 (2004). 
[13] O. Gritsenko, K. Pernal, E. J. Baerends, J. Chem. Phys., 122, 204102 (2005).

[14] N. N. Lathiotakis, N. Helbig, and E. K. U. Gross, Phys. Rev. A 72, 030501 (2005).

[15] J. Cioslowski and K. Pernal, Phys. Rev. B 71, 113103 (2005).

[16] P. Leiva, and M. Piris, J. Chem. Phys., 123, 214102 (2005).

[17] C. Kollmar, J. Chem. Phys., 125, 084108 (2006).

[18] A. Coleman, Rev. Mod. Phys. 35, 668 (1963).

[19] N. Helbig, N. N. Lathiotakis, M. Albrecht, E. K. U. Gross, Europhys. Lett. 77, 67003 (2007).

[20] V. N. Staroverov and G. E. Scuseria, J. Chem. Phys. 117, 2489 (2002).

[21] J. M. Herbert, J. E. Harriman, Chem. Phys. Lett. 382, 142 (2003).

[22] G. Ortiz, Phys. Rev. B 45, 11328 (1992);

[23] C. Filippi, D. J. Singh, C. J. Umrigar, Phys. Rev. B 5014947 (1994);

[24] K. Terakura, T. Oguchi, A. R. Williams, J. Kübler, Phys. Rev. B 304734 (1984);

[25] Sharma S., Dewhurst J. K., and Ambrosch-Draxl C., Phys. Rev. Lett. 95, 136402 (2005).

[26] H. Yasuhara, S. Yoshinaga, and M. Higuchi, Phys. Rev. Lett. 83, 3250 (1999).

[27] J. Cioslowski and K. Pernal, J. Chem. Phys. 111, 3396 (1999).

[28] E. H. Lieb and S. Oxford, Int. J. Quantum Chem. 19, 427 (1981).

[29] G. Ortiz, P. Ballone, Phys. Rev. B50, 1391 (1994); 56, 9970 (1997).

[30] P. Gori-Giorgi and P. Ziesche, Phys. Rev. B66, 235116 (2002).

[31] J. P. Perdew, Y. Wang, Phys. Rev. B 45, 13244 (1992).

[32] D. M. Ceperley and B. J. Alder, Phys. Rev. Lett. 45, 566 (1980). 